\title{
Water mobility along olivine grain boundaries
}

\author{
ADRIEN NÉRI ${ }^{1}$, LISA EBERHARD ${ }^{2}$, MICHIHIKO \\ NAKAMURA $^{3}$ AND DANIEL FROST ${ }^{2}$ \\ ${ }^{1}$ Universität Bayreuth \\ ${ }^{2}$ University of Bayreuth \\ ${ }^{3}$ Graduate School of Science, TOHOKU University Aoba-ku, \\ Sendai 980-8578
}

Presenting Author: adrien.neri@uni-bayreuth.de

Water is an important component within the Earth's mantle and more specifically in the upper mantle due to its solubility in nominally anhydrous upper mantle minerals (NAMs) [e.g. 1]. Addressing the distribution of water throughout the mantle is of prime importance as it can not only cause melting and metasomatism but can also affect the plastic and elastic properties of NAMs and has thus influenced the evolution and present state of the mantle. Water distribution in the mantle depends on the relative efficiency of water diffusivity and mantle dynamics [e.g. 2].

Water can be mobile through two different processes: (1) lattice diffusion and (2) free water migration along grain boundaries. Indirect observation of hydrogen grain boundary diffusion in an olivine matrix indicates that this process is a few orders of magnitude more efficient than lattice diffusion but may become negligible when considering relevant mantle grain sizes [e.g. 3]. Conversely, permeability and percolation velocity should increase with grain size, highlighting a paradox. Here we propose to investigate water mobility along olivine grain boundaries for different grain sizes upon water delivery by the dehydration of cold and dense hydrous subducting slabs. To this aim, we developed a new experimental setup to directly observe diffusion of water along olivine grain boundaries. A sintered olivine core is sandwiched between a water source and a water sink. At high pressure and temperature conditions, the water source dehydrates and releases free water that migrates through the olivine core, via the grain boundaries. The overlying water sink is then progressively hydrated, its degree of hydration reflects the diffusion kinetics, which in turn mainly depends on the olivine core grain size. Time-series experiments show an extremely high efficiency of water mobility along olivine grain boundaries, the water sink starts to be hydrated in a matter of few hours.

[1] J. Ingrin and H. Skogby (2000), European Journal of Mineralogy, 12

[2] G. Richard, M. Monnereau and J. Ingrin (2002), Earth and Planetary Science Letters, 205

[3] S. Demouchy (2010), Earth and Planetary Science Letters, 295 